BULL. AUSTRAL. MATH. SOC.

VOL. $28(1983), 159-160$.

\title{
ON THE EXISTENCE OF EMBEDDED MINIMAL 2-SPHERES IN THE 3-SPHERE, ENDOWED WITH AN ARBITRARY METRIC
}

\author{
Francis R. SMITH
}

In this thesis it is proved that in the 3-sphere endowed with any Riemannian metric (denoted by $N$ ), there exists an embedded, minimal 2-sphere.

Previously, the work of Sacks and Unlenbeck [3] had shown that there exists a stationary (in general, not stable) immersion of $S^{2}$ into $N$. All other results concerning minimal immersions or embeddings of $S^{2}$ into a compact 3-manifold have excluded the case when the ambient space is topologically a 3-sphere.

First, by modifying the minimax techniques of Pitts [2], it is shown that there exists in $N$ a stationary 2-varifold $V$ which can be written as the (varifold) limit of embedded 2-spheres and which has certain local stability properties. Then, using these stability properties together with the recently developed regularity theorems of Almgren and Simon [1] and Schoen, Simon and Yau [4], one can prove that $V$ has the form

$$
V=\sum_{j=1}^{R} n_{j} \underline{\underline{\mathrm{v}}}\left(M_{j}\right) \quad\left(n_{j}, R \in\{\text { integers }\}\right)
$$

where $M_{j}(j=1, \ldots, R)$ are embedded, oriented, connected minimal surfaces in $N$ and where $\underline{\underline{\mathrm{v}}}\left(M_{j}\right) \quad(j=1, \ldots, R)$ denotes the multiplicity

Received 13 April 1983. Thesis submitted to the University of Melbourne, April 1982. Degree approved January 1983. Supervisor: Professor L.M. Simon. 
one varifold associated with $M_{j}$.

Finally, each $M_{j}$ is shown to be diffeomorphic to $S^{2}$. The proof of this makes a more subtle use of the stability properties of $V$, together with the fact that $V$ is the weak limit of embedded 2-spheres. Here it was necessary to make use of some previously unpublished results of simon. The main results of the thesis will be published shortly in a joint paper with Simon.

\section{References}

[1] F. Almgren, Jr. and L.M. Simon, "Existence of embedded solutions to Plateau's problem", Ann. Scuola Norm. Sup. Pisa 6 (1979), 447-495.

[2] J. Pitts, Existence and regularity of minimal surfaces in Riemannian manifolds (Mathematical Notes, 27. Princeton University Press, Princeton, 1981).

[3] J. Sacks and $K$. Unlenbeck, "The existence of minimal immersions of 2-spheres", Ann. of Math. (2) 113 (1981), 1-24.

[4] R. Schoen, L.M. Simon and S.T. Yau, "Curvature estimates for minimal hypersurfaces", Acta Math. 134 (1975), 275-288.

38 Willow Grove,

East Kew,

Victoria 3102,

Australia. 